\title{
Treatment satisfaction and quality of life in patients treated with fingolimod
}

This article was published in the following Dove Press journal:

Patient Preference and Adherence

\author{
Claude Mékiès' \\ Olivier Heinzlef ${ }^{2}$ \\ Béatrice Jenny ${ }^{3}$ \\ Anne-Laure Ramelli ${ }^{4}$ \\ Pierre Clavelou ${ }^{5}$ \\ 'Clinique des Cèdres, Toulouse, \\ ${ }^{2}$ Neurology Department, $\mathrm{CHI}$ Poissy- \\ Saint-Germain-en-Laye, St Germain- \\ en-Laye, ${ }^{3}$ alSacEP, CHU Strasbourg, \\ Strasbourg, ${ }^{4}$ Créteil, ${ }^{5}$ Service de \\ Neurologie, CHU Gabriel Montpied, \\ University of Auvergne, Clermont- \\ Ferrand, France
}

\begin{abstract}
Background: The development of oral treatments for relapsing-remitting multiple sclerosis (RRMS) may alter patient satisfaction and quality of life $(\mathrm{QoL})$. The aim of this survey was to evaluate treatment satisfaction and QoL in patients treated with fingolimod in everyday clinical practice in France.

Methods: Neurologists treating MS in France were invited to participate in the survey by telephone. Each physician was expected to recruit up to six patients with RRMS currently being treated with fingolimod. Enrolled patients were asked to complete the Treatment Satisfaction Questionnaire for Medication (TSQM), the 3-level 5-dimension EuroQoL instrument, as well as specific questions on change in QoL since starting fingolimod. Factors associated with the TSQM score were evaluated using multiple logistic regression analysis.
\end{abstract}

Results: Two hundred and fourteen patients were recruited by 54 neurologists. The mean age of the patients was $41.6 \pm 10.0$ years, and $73.4 \%$ of them were women. During the hospitalization for initiation of fingolimod treatment, $70.1 \%$ of patients had received information on MS, $76.6 \%$ had received information on fingolimod, and $20.7 \%$ had participated in a therapeutic education program. The two variables with the strongest associations with high TSQM scores $(\geq 75)$ were a positive perception of initial hospitalization (hazard ratio: 10.27) and receiving information on MS during hospitalization (hazard ratio: 5.70). The mean EQ-visual analog scale score was 71.6 \pm 16.8 . The mean EQ-visual analog scale score was significantly higher in patients satisfied with their treatment $(75.8 \pm 15.2)$ compared to those unsatisfied with treatment $(66.6 \pm 17.2)$. The proportion of patients who reported an improvement in their capacity to plan for the future was higher in satisfied (72.6\%) than in unsatisfied patients $(49.5 \%)$.

Conclusion: The majority of patients treated with fingolimod are satisfied with their treatment. Treatment satisfaction is associated with better self-rated QoL and an improvement of QoL since starting treatment.

Keywords: multiple sclerosis, quality of life, treatment satisfaction, patient information, fingolmod, EQ-5D, TSQM, therapeutic education

\section{Introduction}

Multiple sclerosis (MS) is a frequent, progressive disabling disease of the central nervous system, generally appearing in young adulthood. In France, the number of individuals affected is believed to be around $100,000 .{ }^{1}$ The disease most frequently presents a relapsing-remitting (RR) course, ${ }^{2}$ in which initial episodic acute exacerbations of neurological disability are associated with slow accumulation of irreversible disability; however, the course varies widely between individuals and is largely unpredictable. ${ }^{3}$ MS carries a high burden of disease in terms of invalidity, psychological distress, quality of life (QoL), and associated cost. ${ }^{4-6}$

Specific disease-modifying treatments (DMTs) for RRMS have been available for over 20 years, and these can reduce the incidence of acute exacerbations and
Correspondence: Claude Mékiès Clinique des Cèdres, Château d'Alliez, Cornebarrieu-CS 20220, 31705

Blagnac Cedex, France

Tel +33562 I3 31 9l

Email c.mekies@wanadoo.fr 
potentially slow progression of disability. The first generation of DMTs, namely, interferon- $\beta$ and glatiramer acetate, are self-administered by subcutaneous or intramuscular injection. However, many studies have demonstrated that adherence, compliance, and persistence with these injectable DMTs are suboptimal, with up to $50 \%$ of treated patients discontinuing regular treatment within 2 years. ${ }^{7-12}$ Important reasons for nonadherence include systemic or local side effects associated with injection ${ }^{7}$ and the constraints associated with self-injection. ${ }^{13}$

More recently, oral DMTs for RRMS have become available, the first of which was fingolimod (since 2011 in France). This agent is indicated as a second-line treatment in patients with persistent active disease in patients taking injectable DMTs correctly and as a first-line treatment in patients with severe, rapidly evolving disease. ${ }^{14}$ Since oral DMTs such as fingolimod and, more recently, dimethylfumarate and teriflunomide do not present the constraints associated with injectable therapies, they may be more acceptable to patients for use over long periods. Indeed, there is some evidence that treatment satisfaction with oral DMTs for MS is higher than that with injectable treatments due to greater convenience of use. ${ }^{15,16}$ Many studies in MS and in other chronic diseases have demonstrated an association between treatment satisfaction and adherence to medication ${ }^{17-19}$ as well as between satisfaction and QoL. ${ }^{20-22}$ In general, determinants of treatment satisfaction with DMTs for MS are poorly understood. Better knowledge of these determinants could provide clues to improving QoL in patients with MS.

The principal objective of this observational survey was to describe satisfaction of patients starting treatment with fingolimod and patient variables associated with treatment satisfaction. Secondary objectives included the evaluation of QoL.

\section{Methods Survey design}

This was a cross-sectional, observational survey conducted in France between August 2015 and January 2016. The survey was implemented by Kantar Health (Paris, France), an international health care market research organization. Six hundred and thirty-two randomly selected neurologists in France were invited to participate by telephone, followed, for physicians who accepted to participate, by a face-to-face visit in order to explain the goals and conduct of the survey and provide the necessary material.

\section{Patients}

Each participating physician was expected to include consecutively up to six patients with RRMS who attended a routine consultation after the start of the survey and who fulfilled the eligibility criteria and agreed to take part in the survey. Eligibility criteria included a diagnosis of RRMS, treatment with fingolimod for at least 6 months, and at least one previous immunodulatory treatment prior to initiation of fingolimod.

\section{Data collection}

Participating physicians provided information on their demographic characteristics and practice and completed a patient case report form during the consultation. A patient auto-questionnaire was given to the patient to be completed in the waiting room at the physician's office. The case report form collected data on the medical history and clinical characteristics of their patients. The patient auto-questionnaire collected information on sociodemographic characteristics, therapeutic education received by the patient, and adverse events. The patient also completed the Treatment Satisfaction Questionnaire for Medication (TSQM) ${ }^{23}$ and the 3-level 5-dimension EuroQoL questionnaire (EQ-5D-3L). ${ }^{24}$ The TSQM consists of ten specific questions grouped into four dimensions (effectiveness, side effects, convenience, and general satisfaction) and a general question about whether the subject experiences side effects. Each specific question is scored on a Likert scale, from which four dimension scores, ranging from 0 to 100 , can be calculated. The EQ-5D-3L includes five dimensions (mobility, self-care, usual activities, pain, and anxiety/depression) that can be rated on three levels of impact (no problems, some problems, and considerable problems). These questions are completed by a $20 \mathrm{~cm}$ visual analog scale (EQ-VAS) on which subjects reply to the following question "I would now like you to tell me the point on this scale where you would put your own health state today." Possible values range from 0 (poorest health state) to 100 (optimal health state). In addition to the EQ-5D, two specific questions were asked relating to perceived change in QoL since starting fingolimod treatment. These were "since starting fingolimod, how would you say that your QoL has evolved?" and "since starting fingolimod, how would you say that your capacity to plan for the future has evolved?" These two questions could be responded to on five-point Likert scales ranging from much worsened to much improved.

\section{Statistical analysis}

The presentation of the data is principally descriptive. Continuous data are presented as mean values \pm standard deviations or median values. Categorical variables are presented as frequency counts with percentages. 
The association between fingolimod treatment duration (dichotomized into $<12$ months and $\geq 12$ months) on the one hand and TSQM general satisfaction score or EQ-VAS score on the other was evaluated using Student's $t$-test. The association between fingolimod treatment duration and TSQM general satisfaction score (dichotomized above and below the mean value into $<75$ and $\geq 75$ ) on the one hand and EQ-5D-3L response distribution on the other was evaluated using the $\chi^{2}$ test. The association between TSQM general satisfaction score and EQ-VAS score was evaluated using Student's $t$-test.

Stepwise multivariate analysis was conducted to identify variables potentially associated with a TSQM general satisfaction score $\geq 75$. In the first step, all variables documented on the patient's auto-questionnaire were assessed for association with the general satisfaction score in a univariate analysis. Those variables for which an association was observed were entered in a backward stepwise multiple regression model, with a probability threshold of 0.05 being required for a variable to be retained in the next step. The variables retained in the final step were then entered into a confirmatory multiple linear regression analysis in order to generate odds ratios. All statistical analyses were performed using SPSS software version 23 (IBM, Armonk, NY, USA).

\section{Ethical considerations}

The survey was conducted in accordance with the ESOMAR International Code on Market and Social Practice, the EphMRA Code of Conduct, relevant current French and European legislation, and Good Epidemiological Practice guidelines. The physicians were required to give a letter of information to their patients, who in turn were asked to provide oral consent to their physician before being able to take part in the survey. The letter of information included a summary of the recommendations of the French Data Protection Authority (Commission Nationale de l'Informatique et des Libertes) on the protection of personal data and the processing of the data from the survey. Using a dedicated hotline, patients also had access to all data concerning them as well as the right to correct or delete such data, in conjunction with Kantar Health. To guarantee complete confidentiality, data collection, processing, and analysis were centralized by Kantar Health.

\section{Results}

\section{Participating neurologists}

Of the 632 neurologists invited to participate in the survey, 54 $(8.5 \%)$ agreed to take part and included at least two patients each. Of these, 26 (48.2\%) were practicing in a hospital setting only, $6(11.1 \%)$ in community practice, and 22 $(40.7 \%)$ in both settings. The mean length of practice was $19.7 \pm 8.1$ years. Seventeen $(31.5 \%)$ of them were women, and $25(46.3 \%)$ were aged over 50 . Every month, they received a mean of $32.9 \pm 28.3$ patients with RRMS for consultation.

\section{Patients}

These 54 neurologists included 214 patients with RRMS and treated with fingolimod. The mean age of the patients was 41.6 years, and 157 (73.4\%) of them were women. Their mean disease duration was $9.0 \pm 6.2$ years and their mean Expanded Disability Status Scale (EDSS) score 2.4 (median: 2). The mean duration of fingolimod treatment was 22.4 months. The demographic and clinical features of the patient population are summarized in Table 1 .

Table I Sociodemographic and clinical characteristics of the survey population ( $\mathrm{N}=2 \mathrm{I}$ )

\begin{tabular}{ll}
\hline Gender (women; $\mathrm{n}(\%))$ & $\mathrm{I} 57(73.4 \%)$ \\
Age (years; mean \pm SD) & $41.6 \pm 10.0$ \\
Marital status & \\
Single & $56(26.2 \%)$ \\
Married/cohabiting & $127(59.3 \%)$ \\
Divorced/Widowed & $30(14.0 \%)$ \\
No answer & $1(0.5 \%)$ \\
Education level & \\
No high school diploma & $22(10.3 \%)$ \\
High school & $94(43.9 \%)$ \\
University/technical college & $96(44.9 \%)$ \\
No answer & $2(0.9 \%)$ \\
Professional activity & \\
In active employment & $117(54.7 \%)$ \\
Invalidity status & $47(22.0 \%)$ \\
Homemaker & $19(8.9 \%)$ \\
Seeking work & $1 \mathrm{I}(5.1 \%)$ \\
Other (including retired and student) & $24(I 1.2 \%)$ \\
No answer & $2(0.9 \%)$ \\
Time since diagnosis (years; mean \pm SD) & $9.0 \pm 6.2$ \\
MS relapses in the previous I2 months (mean \pm SD) & $1.5 \pm 0.9$ \\
EDSS score at inclusion (mean \pm SD) & $2.4 \pm 1.7$ \\
Treatment history & $\mathrm{N}=204$ \\
Duration of fingolimod treatment (in months) & $22.4 \pm 12.5$ \\
Number of previous first-line treatments & \\
I & $135(63.1 \%)$ \\
2 & $63(29.4 \%)$ \\
No & $11(5.1 \%)$ \\
Previous first-line treatment ${ }^{\mathrm{a}}$ & $5(2.3 \%)$ \\
Interferon- $\beta$ & \\
Glatiramer acetate & $138(64.5 \%)$ \\
Dimethylfumarate & $72(33.6 \%)$ \\
No answer & $9(4.2 \%)$ \\
\hline Noter & $1(0.5 \%)$ \\
\hline
\end{tabular}

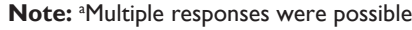

Abbreviations: EDSS, Expanded Disability Status Scale; MS, multiple sclerosis; SD, standard deviation. 


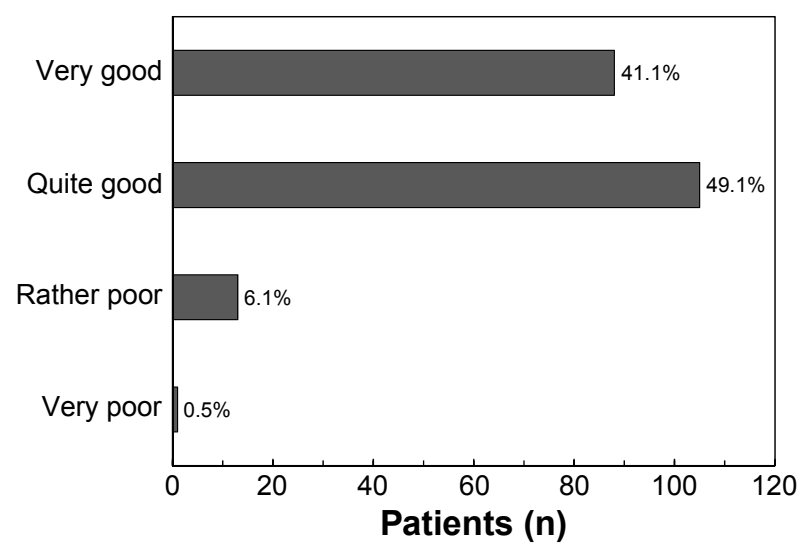

Figure I Timing and content of therapeutic education programs. Note: Data were missing for five patients.

\section{Patient information}

Patients starting fingolimod treatment are required to take the first dose under supervision in hospital over an observation period of 6 hours. One hundred and ninety-three (90.2\%) patients reported that their experience of this hospitalization was very or quite good (Figure 1). During this hospitalization, $164(76.6 \%)$ patients had the opportunity to discuss their treatment with hospital staff (Table 2). However, only $44(20.6 \%)$ patients had participated in a formal therapeutic education program, 37 on MS and 29 on fingolimod treatment. Of the remaining 168 patients, $59(35.1 \%)$ had received no further information on their disease or treatment from health care professionals. Around half the patients had sought information about fingolimod on the internet.

\section{Treatment satisfaction}

The mean TSQM global satisfaction score was $74.2 \pm 16.3$ (median: 68.8), with the highest individual dimension score being observed for the side effects dimension 87.6 \pm 18.2 (median: 92.4) (Figure 2). The mean effectiveness score was 67.1 \pm 20.0 (median: 64.5), and the mean convenience score

Table 2 Patient information ( $\mathrm{N}=2 \mid 2)$

\begin{tabular}{ll}
\hline Opportunity to discuss MS with hospital staff & $150(70.1 \%)$ \\
Opportunity to discuss treatment with hospital staff & $164(76.6 \%)$ \\
Participation in a therapeutic education program about MS & $51(23.8 \%)$ \\
Participation in a therapeutic education program about & $44(20.6 \%)$ \\
fingolimod & \\
Performed internet searches about fingolimod & $124(58.0 \%)$ \\
Before starting treatment & $102(47.7 \%)$ \\
After starting treatment & $56(26.2 \%)$ \\
About efficacy & $101(47.2 \%)$ \\
About potential side effects & $95(44.4 \%)$ \\
About other patients' experience & $50(23.4 \%)$ \\
\hline
\end{tabular}

Abbreviation: MS, multiple sclerosis.

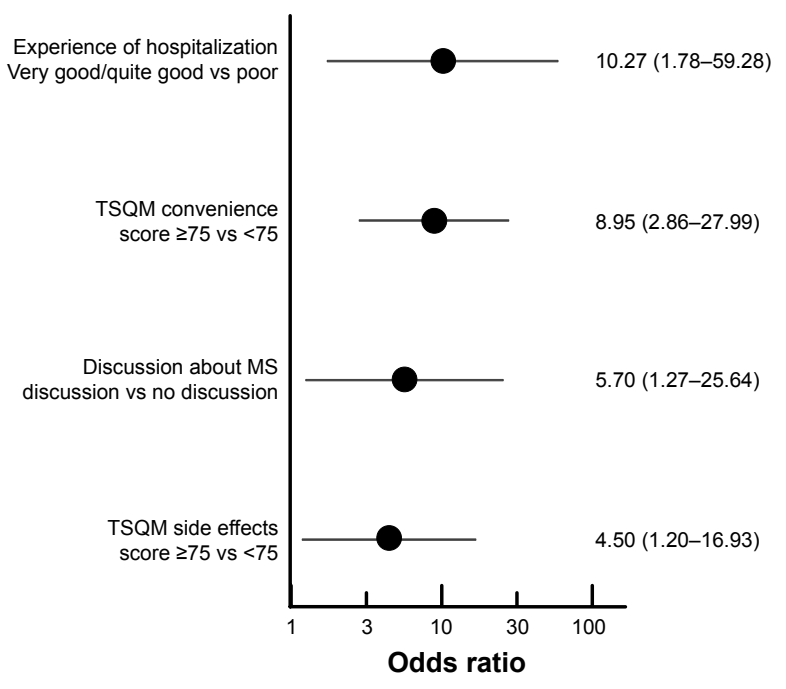

Figure 2 Variables associated with a TSQM general satisfaction score $\geq 75$ vs $<75$. Note: Data are represented as odds ratios with their $95 \%$ confidence intervals. Abbreviations: MS, multiple sclerosis; TSQM, Treatment Satisfaction Questionnaire for Medication.

78.4 \pm 20.1 (median: 78.9). No difference in any of the four dimension scores was observed between patients who had started treated with fingolimod within the previous 12 months and those treated for longer periods. The proportion of patients with a mean TSQM global satisfaction score $\geq 75$ did not differ significantly between patients with an EDSS score $\leq 3(54.5 \%)$ and those with a score $>3(50.0 \%)$. With respect to the side effects question of the TSQM, 63 patients $(29.4 \%)$ reported experiencing side effects. The most frequently reported were fatigue (24 patients; $11.2 \%$ ), headache or dizziness (11 patients; 5.1\%), and gastrointestinal disturbances (7 patients; $3.3 \%$ ).

In the multivariate logistic regression analysis, four variables were found to be independently associated with a TSQM general satisfaction score $\geq 75$ (Figure 2), namely, a very good perception of the initial hospitalization (odds ratio: 10.27) and having the opportunity to discuss MS with hospital staff during hospitalization for initiation of fingolimod (odds ratio: 5.70), as well as two of the constituent dimensions of the TSQM itself (side effects and convenience).

\section{QoL}

The distribution of the three different response modalities for the five dimensions of the EQ-5D is presented in Figure 3. The most impacted dimensions were pain/discomfort (143 patients reporting problems; 66.8\%), anxiety/depression (130 patients; 60.7\%), and mobility (101 patients; $47.2 \%$ ). The mean EQ-VAS score was 71.6 \pm 16.8 (median: 74.8). 


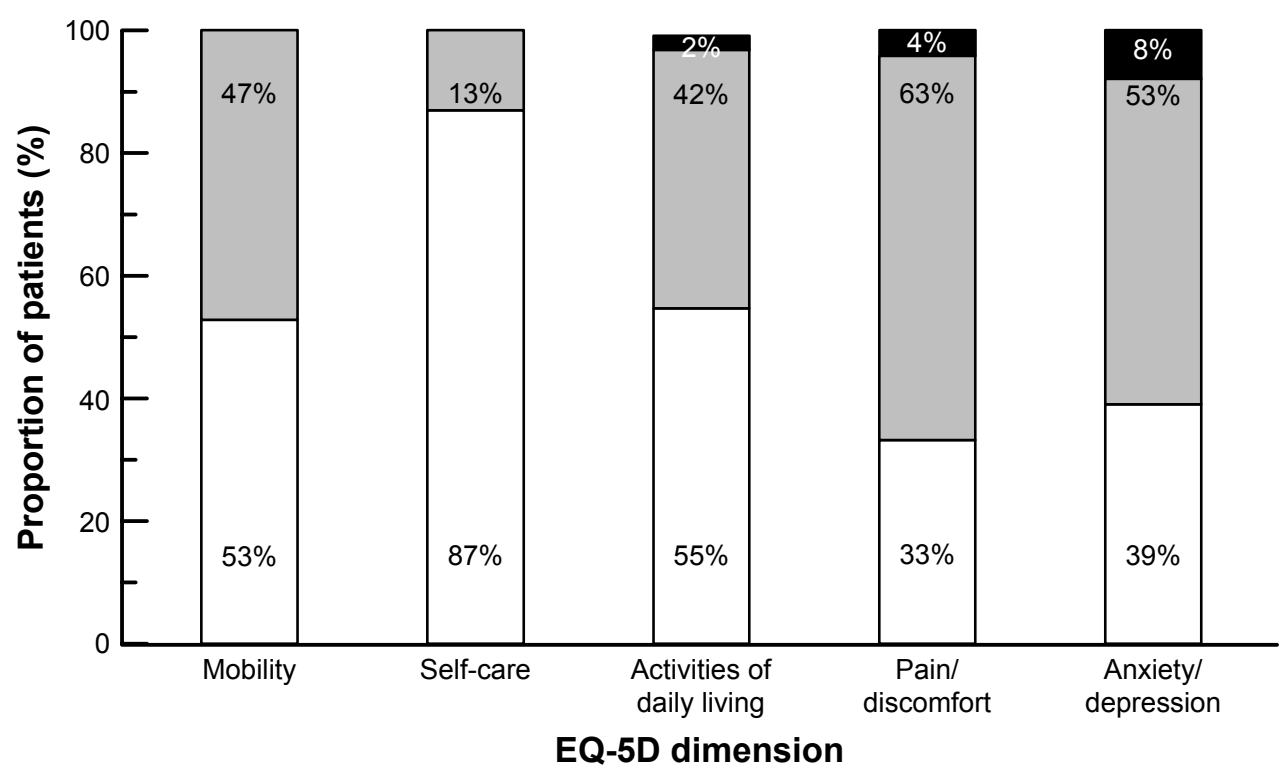

Figure 3 Distribution of response modalities for the EQ-5D.

Notes: Open segments, no problems; gray segments, some problems; black segments, considerable problems. Data on activities of daily living were missing for one patient. Abbreviations: EQ-5D, 5-dimension EuroQoL questionnaire; QoL, quality of life.

No significant difference in response distribution was observed according to the duration of fingolimod treatment ( $<12$ or $\geq 12$ months) for any of the dimensions of the EQ-5D, nor in the EQ-VAS score. In contrast, higher
EQ-VAS scores were strongly associated with an EDSS score $>3$ ( $p<0.01 ; \chi^{2}$-test; Figure 4$)$.

In addition, the proportion of patients reporting problems was significantly lower for all EQ-5D dimensions except

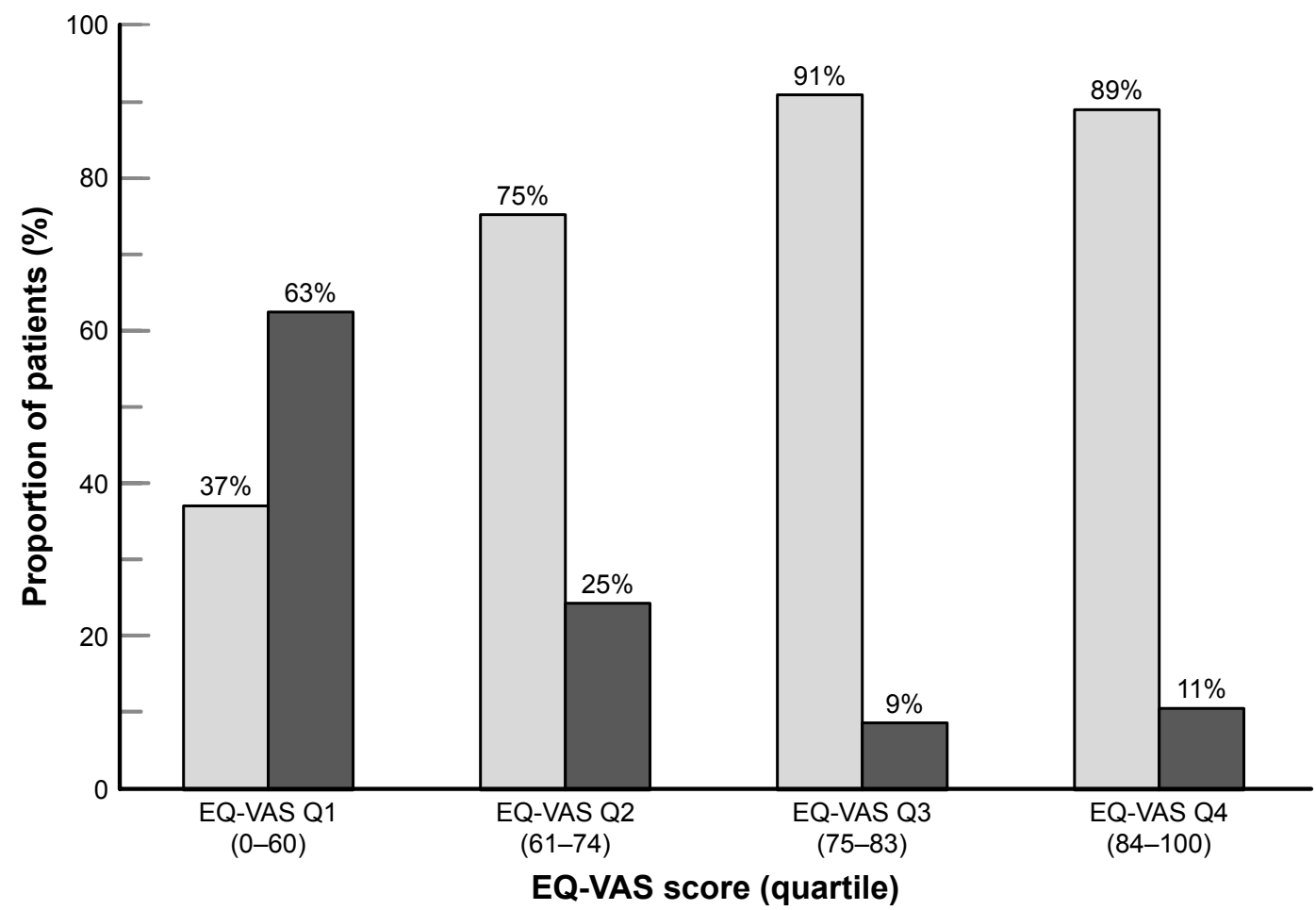

Figure 4 Distribution of EQ-VAS scores as a function of EDSS disability score.

Notes: Light segments, EDSS score $\leq 3(\mathrm{~N}=158)$; dark segments, EDSS score $>3(\mathrm{~N}=56)$. EQ-VAS scores are divided into quartiles: Ist quartile: $0-60$; 2 nd quartile: $61-74$; 3 rd quartile: $75-83$; 4th quartile: 84-100.

Abbreviations: EDSS, Expanded Disability Status Scale; EQ-VAS, EuroQol visual analog scale; QoL, quality of life. 


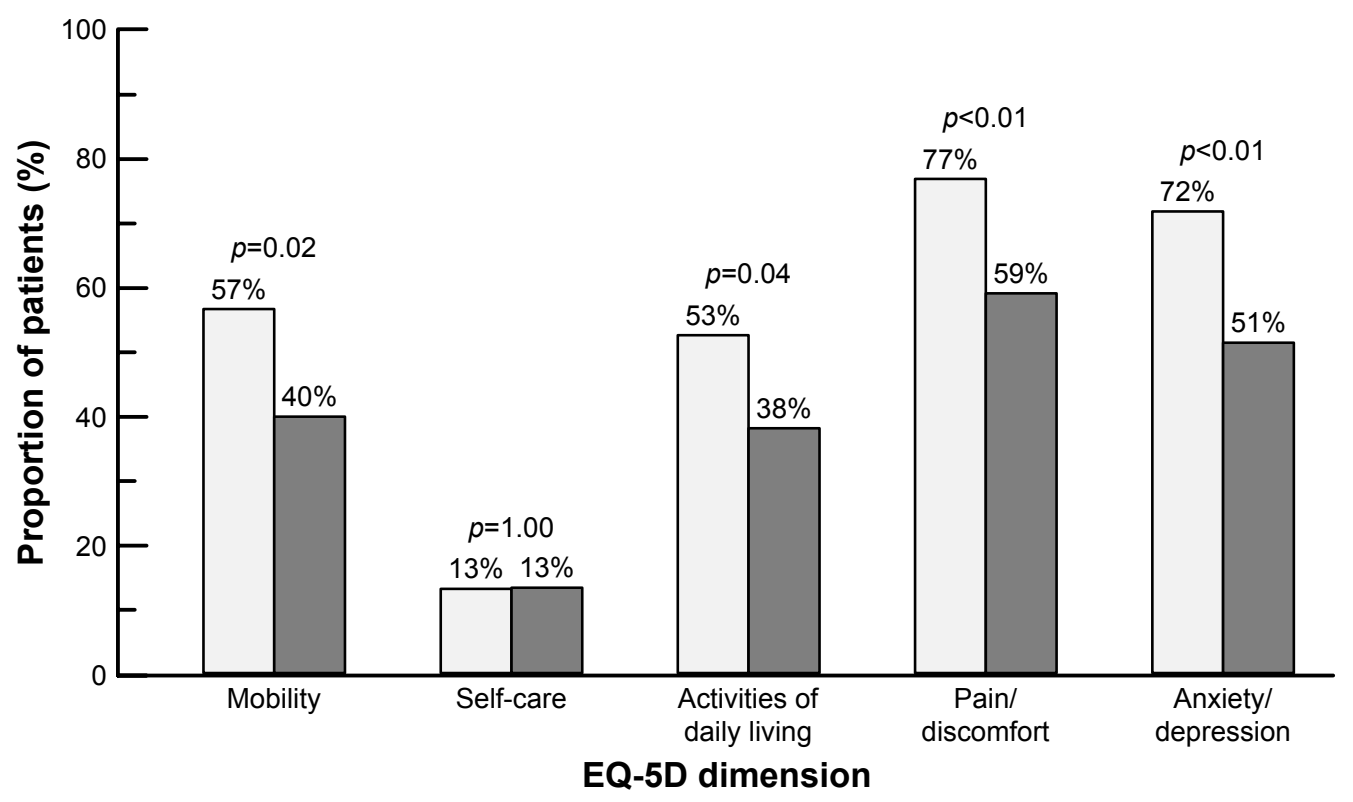

Figure 5 Distribution of TSQM response modalities for the EQ-5D.

Notes: Data are presented as the proportion of patients reporting some or considerable problems on each dimension of the EQ-5D. Light columns, TSQM general satisfaction score $\geq 75$ ( $N=1$ I3); dark columns, TSQM general satisfaction score $<75$ ( $N=99$ ).

Abbreviations: EQ-5D, 5-dimension EuroQoL questionnaire; TSMQ, Treatment Satisfaction Questionnaire for Medication; QoL, quality of life.

self-care in patients who were satisfied with their treatment (TSQM general satisfaction score $\geq 75$ ) than in patients who were not (Figure 5). Similarly, the EQ-VAS score was significantly higher in the more satisfied patients $(75.8 \pm 15.2$ vs $66.6 \pm 17.2 ; p<0.01$; Student's $t$-test).

With respect to the three specific questions on change in QoL (Table 3), 72.4\% of patients considered that their QoL in general was much or somewhat improved since starting fingolimod, $62.1 \%$ considered that their capacity to plan for the future was much or somewhat improved, and $49.5 \%$ considered that their confidence in the future was much or somewhat improved. The proportion of patients who considered that these aspects had changed for the better was significantly higher in patients who were satisfied with their treatment (TSQM general satisfaction score $\geq 75$ ) compared to those who were not $(p<0.05$ in each case).

\section{Discussion}

The goal of this survey was to estimate treatment satisfaction with fingolimod and to identify variables associated with satisfaction. General satisfaction with treatment was relatively high, and the principal variables associated with high satisfaction were the experience of the hospitalization for the first dose of fingolimod and the opportunity to discuss MS with hospital staff.

The association between treatment satisfaction and these "relationship" variables is a strong one. The fact that only one-quarter of patients claimed to have participated in a therapeutic education program and one-quarter never to have had an opportunity to discuss their disease or its treatment with hospital staff suggests that there is considerable room for improvement in building awareness about MS and its treatment through a constructive dialogue between health care personnel and the patient. The obligation for patients to take their first dose of fingolimod under supervision in hospital provides an opportunity to provide them with information about their treatment and to discuss their expectations and concerns. The need for a constructive and egalitarian patient-physician dialogue in order to optimize adherence and outcome has long been recognized in the

Table 3 Evolution of QoL since starting fingolimod $(\mathrm{N}=2 \mathrm{I} 2)$

\begin{tabular}{|c|c|c|c|c|c|c|}
\hline & \multicolumn{3}{|c|}{ Much or somewhat improved } & \multicolumn{3}{|c|}{ Much or somewhat worsened } \\
\hline & $\overline{\text { All }}$ & TSQM $\geq 75$ & TSQM $<75$ & $\overline{\text { All }}$ & TSQM $\geq 75$ & TSQM $<75$ \\
\hline QoL in general & I53 (72.2\%) & $92(81.4 \%)$ & $61(61.6 \%)$ & $13(6.1 \%)$ & $3(2.7 \%)$ & $10(10.1 \%)$ \\
\hline Capacity to plan for the future & $|3|(6 \mid .8 \%)$ & $82(72.6 \%)$ & 49 (49.5\%) & 14 (6.6\%) & $2(1.8 \%)$ & $12(12.1 \%)$ \\
\hline Confidence in the future & $105(49.5 \%)$ & $67(59.3 \%)$ & $38(38.4 \%)$ & $28(13.2 \%)$ & $9(8 \%)$ & $19(19.2 \%)$ \\
\hline
\end{tabular}

Abbreviations: TSQM, Treatment Satisfaction Questionnaire for Medication; QoL, quality of life. 
management of other chronic diseases such as diabetes. ${ }^{25}$ In MS, treatment adherence has been shown to be better in patients who consider themselves well informed. ${ }^{26} \mathrm{~A}$ recent large international survey has also investigated the relationship between the patient-health care professional relationship and treatment satisfaction in 928 treated patients with RRMS. ${ }^{27}$ In this survey, patients who considered that they had an open dialogue with their neurologist and those who considered that they were involved in treatment decisions reported greater satisfaction with their treatment than patients who did not.

In spite of structured therapeutic education programs that have been established in some MS centers in France, this does not appear to be the norm for all patients. When such programs do exist and their performance has been evaluated, they were considered relevant and useful by patients and were associated with high rates of appropriate and sustained medication use. ${ }^{28}$ The MS in the 21 st Century Steering Group has recently published recommendations on how to improve patient engagement and empowerment, which emphasizes the importance of patient information and awareness. ${ }^{29}$

Several previous studies have been performed with the TSQM in MS, although the different survey designs and versions of the questionnaire used make comparison difficult. ${ }^{30}$ These include the COMPLIANCE survey in Spain, ${ }^{31}$ the THEPA-MS study in Germany, ${ }^{32}$ and an American observational study of patients treated with fingolimod..$^{33}$ These studies reported TSQM general satisfaction scores ranging from 69.8 to 72.6, and our findings (mean general satisfaction score of 74.2) are consistent with these previous reports. Very limited information is available in the literature on the variables associated with treatment satisfaction in patients with MS. Hanson et $\mathrm{al}^{33}$ reported that satisfaction scores were higher in patients who had switched to fingolimod from another DMT compared to those starting fingolimod as a first-line treatment. In our survey, the only patient variables that could be identified as being associated with satisfaction were related to the interaction between the patient and the health care system. Similarly, the multinational study referred to earlier ${ }^{27}$ also found that communication between the patient and the neurologist was an important determinant of treatment satisfaction. Somewhat surprisingly, none of the sociodemographic variables (eg, age or education level) or clinical variables (eg, duration or severity of the disease) was significantly associated with general satisfaction, although such clinical variables have previously been reported to be associated with treatment satisfaction in other studies. ${ }^{32}$ Possible explanations for this difference include the lower power of our survey to detect associated variables with relatively small effect sizes, the strong effects of the "relationship" variables in our survey that may have masked smaller treatment effects, or real differences in determinants of treatment satisfaction between different DMTs. With respect to disease severity, it should be noted that the patients were not particularly disabled (median EDSS score: 2), which may help explain the absence of an association with treatment satisfaction. It is also possible that the reasons that patients switched from a first-line treatment may influence treatment satisfaction with fingolimod. For example, patients who requested to be switched to an oral treatment may be more satisfied than those who switched on the advice of the physician. In this survey, the reasons for the switch were not documented, and thus, it is not possible to address this issue. Since there is little information available in the literature, this potential association would merit investigation in future studies.

Although there was no control group in this survey, the observed EQ-VAS scores in our survey were 10 points lower than the reference values for the French general population (mean score $=81.7) .{ }^{34}$ There is no reference directory of EQ-VAS scores in patients with different chronic diseases in France. However, the mean EQ-VAS scores observed in our treated MS patients are similar to those reported in French studies of patients with uncontrolled asthma, ${ }^{35}$ patients with diabetes experiencing hypoglycemia, ${ }^{36}$ or patients with deep infiltrating endometriosis. ${ }^{37}$ The EQ-VAS scores observed here are relatively close to those reported in a North American registry of patients treated with fingolimod. ${ }^{38} \mathrm{We}$ observed a strong negative association between QoL determined with the EQ-VAS and with disability measured with the EDSS, consistent with previous studies with this ${ }^{39-41}$ and other ${ }^{6,42}$ measures of QoL. The relationship between treatment satisfaction and QoL has previously been demonstrated in other pathologies, including hypertension, ${ }^{20}$ diabetes, ${ }^{21}$ and allergic rhinitis, ${ }^{22}$ and is to be expected. However, this relationship has not been demonstrated before, to our knowledge, in MS. The difference in EQ-VAS scores between satisfied and unsatisfied patients is of similar magnitude to the difference between MS patients and the general population.

We found that the majority of patients reported that their QoL had improved since starting treatment with fingolimod and that their capacity to plan for the future had improved. These perceptions were more frequent in patients who were satisfied with their treatment than in those who were not. Although these data were not collected prospectively, the findings are consistent with those of other interventional ${ }^{43,44}$ or observational ${ }^{45}$ studies that have also reported improvements in health-related QoL in the months following 
initiation of fingolimod treatment. These findings highlight the interest of switching patients to fingolimod who do not respond satisfactorily to a first-line therapy in order to improve treatment satisfaction, QoL, and, potentially, treatment adherence.

\section{Limitations}

This survey has a number of strengths and limitations. The strengths include the range of variables documented and the very limited extent of missing data. Some inclusion bias may arise from the eligibility criterion necessitating that patients be treated with fingolimod for at least 6 months before inclusion. It is possible that patients who were dissatisfied with treatment or had experienced problems, such as side effects, may have discontinued treatment before 6 months, leading to an overrepresentation of satisfied patients in our sample. In addition, since this was a cross-sectional survey, the information on how QoL evolved over time is derived from a retrospective patient self-report and is thus open to recall bias. A similar such bias may apply to the data on the information or education received by patients, which is also documented retrospectively. Finally, since participation was voluntary, it cannot be excluded that neurologists who were more interested in issues related to patient education or patients who were more satisfied with their treatment were more likely to participate in the survey, which may have led to some bias.

\section{Conclusion}

The majority of patients treated with fingolimod are satisfied with their treatment. Treatment satisfaction is associated with better self-rated QoL and a perceived improvement of QoL since starting treatment. The more patients are informed on the disease and its treatments, the higher the level of treatment satisfaction that they express. Higher treatment satisfaction would be expected to translate into better QoL and adherence to treatment. For this reason, treating neurologists need to be aware of the importance of developing a dialogue with patients on knowledge and expectations about their disease and its treatment. In the case of treatment with fingolimod, hospitalization for the first dose provides an opportunity to engage in such a dialogue.

\section{Acknowledgment}

The survey was initiated and financially supported by Novartis. Operational implementation of the survey was delegated by the sponsor to Kantar Health (Paris, France), which was responsible for recruiting participating physicians and data collection and analysis. Editorial support for the preparation of this paper was provided by SARL Foxymed (Paris, France).

\section{Disclosure}

$\mathrm{CM}$ has received consultancy fees or speaking fees from Novartis, Biogen, Bayer, Merck, Teva, Genzyme/Sanofi, Allergan, Almirall, EISAI, Coloplast, Lundbeck, and Pfizer. PC has received consultancy fees from Almirall, Bayer Health, Biogen, Genzyme-Sanofi, Merck, Novartis, and Teva Pharma. $\mathrm{OH}$ has received consultancy fees from Genzyme, Novartis, and Teva Pharma. BJ has received consultancy fees from Bayer Health, Biogen, Merck, Novartis, and Teva Pharma. ALR has received consultancy fees from Novartis. The authors constituted the scientific advisory board of the survey, established by the survey sponsor in order to advise on the design of the survey and on the analysis and exploitation of the data. The authors report no other conflicts of interest in this work.

\section{References}

1. Foulon S, Maura G, Dalichampt M, et al. Prevalence and mortality of patients with multiple sclerosis in France in 2012: a study based on French health insurance data. J Neurol. 2017;264(6):1185-1192.

2. Lublin FD, Reingold SC, Cohen JA, et al. Defining the clinical course of multiple sclerosis: the 2013 revisions. Neurology. 2014;83(3):278-286.

3. Compston A, Coles A. Multiple sclerosis. Lancet. 2008;372(9648): 1502-1517.

4. Ivanova JI, Birnbaum HG, Samuels S, Davis M, Phillips AL, Meletiche D. The cost of disability and medically related absenteeism among employees with multiple sclerosis in the US. Pharmacoeconomics. 2009; 27(8):681-691.

5. Michalski D, Liebig S, Thomae E, Singer S, Hinz A, Bergh FT. Anxiety, depression and impaired health-related quality of life are therapeutic challenges in patients with multiple sclerosis. Mental Illness. 2010;2(1):e5.

6. Clavelou P, Auclair C, Taithe F, Gerbaud L. Quality of life in multiple sclerosis: theoretical and practical aspects. Rev Neurologique. 2009; 165(Spec No 2):F115-F124.

7. Giovannoni G, Southam E, Waubant E. Systematic review of diseasemodifying therapies to assess unmet needs in multiple sclerosis: tolerability and adherence. Mult Scler. 2012;18(7):932-946.

8. Bonafede MM, Johnson BH, Wenten M, Watson C. Treatment patterns in disease-modifying therapy for patients with multiple sclerosis in the United States. Clin Ther. 2013;35(10):1501-1512.

9. Jokubaitis VG, Spelman T, Lechner-Scott J, et al. The Australian Multiple Sclerosis (MS) immunotherapy study: a prospective, multicentre study of drug utilisation using the MSBase platform. PLoS One. 2013;8(3):e59694.

10. Reynolds MW, Stephen R, Seaman C, Rajagopalan K. Persistence and adherence to disease modifying drugs among patients with multiple sclerosis. Curr Med Res Opin. 2010;26(3):663-674.

11. Zhornitsky S, Greenfield J, Koch MW, et al. Long-term persistence with injectable therapy in relapsing-remitting multiple sclerosis: an 18-year observational cohort study. PLoS One. 2015;10(4):e0123824.

12. Tremlett HL, Oger J. Interrupted therapy: stopping and switching of the beta-interferons prescribed for MS. Neurology. 2003;61(4):551-554.

13. Devonshire V, Lapierre Y, Macdonell R, et al. The Global Adherence Project (GAP): a multicenter observational study on adherence to disease-modifying therapies in patients with relapsing-remitting multiple sclerosis. Eur J Neurol. 2011;18(1):69-77. 
14. Scott LJ. Fingolimod: a review of its use in the management of relapsingremitting multiple sclerosis. CNS Drugs. 2011;25(8):673-698.

15. Vermersch P, Czlonkowska A, Grimaldi LM, et al. Teriflunomide versus subcutaneous interferon beta-1a in patients with relapsing multiple sclerosis: a randomised, controlled phase 3 trial. Multi Scler. 2014;20(6):705-716

16. Calkwood J, Cree B, Crayton H, et al. Impact of a switch to fingolimod versus staying on glatiramer acetate or beta interferons on patient- and physician-reported outcomes in relapsing multiple sclerosis: post hoc analyses of the EPOC trial. BMC Neurol. 2014;14:220.

17. Barbosa CD, Balp MM, Kulich K, Germain N, Rofail D. A literature review to explore the link between treatment satisfaction and adherence, compliance, and persistence. Patient Prefer Adher. 2012;6:39-48.

18. Albrecht G, Hoogstraten J. Satisfaction as a determinant of compliance. Community Dent Oral Epidemiol. 1998;26(2):139-146.

19. Glanz BI, Musallam A, Rintell DJ, Chitnis T, Weiner HL, Healy BC. Treatment satisfaction in multiple sclerosis. Int JMS Care. 2014;16(2): 68-75.

20. Al-Jabi SW, Zyoud SH, Sweileh WM, et al. Relationship of treatment satisfaction to health-related quality of life: findings from a crosssectional survey among hypertensive patients in Palestine. Health Expect. 2015;18(6):3336-3348.

21. Nicolucci A, Cucinotta D, Squatrito S, et al. Clinical and socio-economic correlates of quality of life and treatment satisfaction in patients with type 2 diabetes. Nutr Metab Cardiovasc Dis. 2009;19(1):45-53.

22. Turner RR, Testa MA, Hayes JF, Su M. Validation of the allergic rhinitis treatment satisfaction and preference scale. Allergy Asthma Proc. 2013;34(6):551-557

23. Atkinson MJ, Sinha A, Hass SL, et al. Validation of a general measure of treatment satisfaction, the Treatment Satisfaction Questionnaire for Medication (TSQM), using a national panel study of chronic disease. Health Qual Life Outcomes. 2004;2:12.

24. EuroQol Group. EuroQol - a new facility for the measurement of health-related quality of life. Health Policy. 1990;16(3):199-208.

25. Golin CE, DiMatteo MR, Gelberg L. The role of patient participation in the doctor visit. Implications for adherence to diabetes care. Diabetes Care. 1996;19(10):1153-1164.

26. de Seze J, Borgel F, Brudon F. Patient perceptions of multiple sclerosis and its treatment. Patient Prefer Adher. 2012;6:263-273.

27. Tintoré $\mathrm{M}$, Alexander M, Costello K, et al. The state of multiple sclerosis current insight into the patient/health care provider relationship, treatment challenges, and satisfaction. Patient Prefer Adher. 2017;11:33-45.

28. Colpaert MH. A therapeutic education programme for patients with multiple sclerosis. Multi Scler. 2010;16(Supp1):S170-S171.

29. Rieckmann P, Boyko A, Centonze D, et al. Achieving patient engagement in multiple sclerosis: a perspective from the multiple sclerosis in the $21 \mathrm{st}$ Century Steering Group. Multi Scler Relat Disord. 2015;4(3):202-218.

30. Ting J, Liu Y, Petrillo J, Giannattasio G, Sabatella G. Treatment satisfaction with disease modifying therapies in multiple sclerosis: a systematic review of studies using the Treatment Satisfaction Questionnaire for Medication (Tsqm). Value Health. 2015;18(7):A760-A761.
31. Saiz A, Mora S, Blanco J. Therapeutic compliance of first line diseasemodifying therapies in patients with multiple sclerosis. COMPLIANCE Study. Neurologia. 2015;30(4):214-222.

32. Haase R, Kullmann JS, Ziemssen T. Therapy satisfaction and adherence in patients with relapsing-remitting multiple sclerosis: the THEPA-MS survey. Ther Adv Neurol Disord. 2016;9(4):250-263.

33. Hanson KA, Agashivala N, Stringer SM, Balantac Z, Brandes DW. A cross-sectional survey of patient satisfaction and subjective experiences of treatment with fingolimod. Patient Prefer Adher. 2013;7:309-318.

34. Perneger TV, Combescure C, Courvoisier DS. General population reference values for the French version of the EuroQol EQ-5D health utility instrument. Value Health. 2010;13(5):631-635.

35. Doz M, Chouaid C, Com-Ruelle L, et al. The association between asthma control, health care costs, and quality of life in France and Spain. BMC Pulm Med. 2013;13:15.

36. Vexiau P, Mavros P, Krishnarajah G, Lyu R, Yin D. Hypoglycaemia in patients with type 2 diabetes treated with a combination of metformin and sulphonylurea therapy in France. Diabetes Obes Metab. 2008; 10(Suppl 1):16-24.

37. Touboul C, Amate P, Ballester M, Bazot M, Fauconnier A, Darai E. Quality of life assessment using EuroQOL EQ-5D questionnaire in patients with deep infiltrating endometriosis: the relation with symptoms and locations. Int J Chronic Dis. 2013;2013:452134.

38. Hersh CM, Hara-Cleaver C, Rudick RA, Cohen JA, Bermel RA, Ontaneda D. Experience with fingolimod in clinical practice. Int $J$ Neurosci. 2015;125(9):678-685.

39. Ahmad H, Taylor BV, van der Mei I, et al. The impact of multiple sclerosis severity on health state utility values: evidence from Australia. Multi Scler. 2017;23(8):1157-1166

40. Orme M, Kerrigan J, Tyas D, Russell N, Nixon R. The effect of disease, functional status, and relapses on the utility of people with multiple sclerosis in the UK. Value Health. 2007;10(1):54-60.

41. Reese JP, Wienemann G, John A, et al. Preference-based health status in a German outpatient cohort with multiple sclerosis. Health Qual Life Outcomes. 2013;11:162.

42. Mitchell AJ, Benito-Leon J, Gonzalez JM, Rivera-Navarro J. Quality of life and its assessment in multiple sclerosis: integrating physical and psychological components of wellbeing. Lancet Neurol. 2005; 4(9):556-566.

43. Montalban X, Comi G, O'Connor P, et al. Oral fingolimod (FTY720) in relapsing multiple sclerosis: impact on health-related quality of life in a phase II study. Multi Scler. 2011;17(11):1341-1350.

44. Calabresi PA, Radue EW, Goodin D, et al. Safety and efficacy of fingolimod in patients with relapsing-remitting multiple sclerosis (FREEDOMS II): a double-blind, randomised, placebo-controlled, phase 3 trial. Lancet Neurol. 2014;13(6):545-556.

45. Fox E, Edwards K, Burch G, et al. Outcomes of switching directly to oral fingolimod from injectable therapies: Results of the randomized, openlabel, multicenter, Evaluate Patient OutComes (EPOC) study in relapsing multiple sclerosis. Multi Scler Relat Disord. 2014;3(5):607-619.
Patient Preference and Adherence

\section{Publish your work in this journal}

Patient Preference and Adherence is an international, peer-reviewed, open access journal that focuses on the growing importance of patient preference and adherence throughout the therapeutic continuum. Patient satisfaction, acceptability, quality of life, compliance, persistence and their role in developing new therapeutic modalities and compounds to optimize

\section{Dovepress}

clinical outcomes for existing disease states are major areas of interest for the journal. This journal has been accepted for indexing on PubMed Central. The manuscript management system is completely online and includes a very quick and fair peer-review system, which is all easy to use. Visit http://www. dovepress.com/testimonials.php to read real quotes from published authors. 